\title{
Phacomatosis spilorosea versus phacomatosis melanorosea: a critical reappraisal of the worldwide literature with updated classification of phacomatosis pigmentovascularis
}

\author{
Daniele Torchia ${ }^{1 凶}$ \\ ${ }^{1}$ Department of Dermatology, James Paget University Hospital, Gorleston-on-Sea, United Kingdom.
}

\begin{abstract}
Introduction: Phacomatosis pigmentovascularis is a term encompassing a group of disorders characterized by the coexistence of a segmental pigmented nevus of melanocytic origin and segmental capillary nevus. Over the past decades, confusion over the names and definitions of phacomatosis spilorosea, phacomatosis melanorosea, and their defining nevi, as well as of unclassifiable phacomatosis pigmentovascularis cases, has led to several misplaced diagnoses in published cases.

Methods: A systematic and critical review of the worldwide literature on phacomatosis spilorosea and phacomatosis melanorosea was carried out.

Results: This study yielded 18 definite instances of phacomatosis spilorosea and 14 of phacomatosis melanorosea, with one and six previously unrecognized cases, respectively.

Conclusions: Phacomatosis spilorosea predominantly involves the musculoskeletal system and can be complicated by neurological manifestations. Phacomatosis melanorosea is sometimes associated with ancillary cutaneous lesions, displays a relevant association with vascular malformations of the brain, and in general appears to be a less severe syndrome. Established phacomatosis pigmentovascularis variants now include phacomatosis cesioflammea, phacomatosis cesiomarmorata, phacomatosis spilorosea, phacomatosis melanorosea, phacomatosis cesioflammeomarmorata, and phacomatosis melanocesioflammea.
\end{abstract}

Keywords: classification, mosaicism, phacomatosis melanorosea, phacomatosis pigmentovascularis, phacomatosis spilorosea, review

Received: 14 December 2020 | Returned for modification: 24 January 2021 | Accepted: 29 January 2021

\section{Introduction}

Phacomatosis pigmentovascularis (PPV) is a term encompassing a group of disorders characterized by the coexistence of a segmental pigmented nevus of melanocytic origin and segmental capillary nevus. Two main classifications have been followed to date, one initiated by Ota et al. in 1947 (1) and progressively updated up to 2003 to include five types (2), and one by Happle in 2005 (3), updated in 2012 to include four variants (4). The latter is now firmly established as the more appropriate one. Phacomatosis cesioflammea (nevus cesius [NC] associated with nevus flammeus) is by far the most commonly observed type, with hundreds of cases reported to date (5). Phacomatosis cesiomarmorata (NC associated with cutis marmorata telangiectatica congenita) is probably the second most common PPV, as testified by a couple of dozen cases identified in reviews that considered PubMed ${ }^{\circledR}$ only $(5,6)$. The remaining two recognized PPV types are characterized by nevus roseus (NR), associated with either a macular nevus spilus (MNS) (phacomatosis spilorosea [PSR]) or a flag-like hypermelanotic nevus (FHN) (phacomatosis melanorosea [PMR]) (7). It was suggested that patients not fitting the above definitions be provisionally placed in the "wastebasket" term of "unclassifiable PPV" (3). Over the past decades, confusion over the names and definitions of PSR, PMR, and their defining nevi has led to several misplaced diagnoses in published cases.

\section{Methods}

A systematic review of the worldwide literature up to Novem- ber 2020 was carried out, using PubMed®, Embase ${ }^{\circledR}$, Scopus $\AA$, Google Scholar, and Global Index Medicus as primary tools. A wide range of keywords were used, including old and new terms identifying the entities reviewed and their defining nevi. A review of the references of every paper retrieved was also performed. A case-by-case, critical reassessment of all cases retrieved was carried out, looking for the presence of 1) a pale pink, flag-like capillary nevus (FCR) and a flag-like melanotic patch with superimposed, densely packed, uniformly distributed, flat melanocytic nevi (MNS) for the diagnosis of PSR (Fig. 1A); or 2) a NR and a flaglike hypermelanotic patch (FHN) for the diagnosis of PMR (Fig. 1B).

\section{Results}

This analysis yielded 18 cases of definite PSR (Table 1) (8-25) and 14 cases of definite PMR (Table 2) (26-39). Patients mentioned in abstracts or in large case series were not included because of a lack of sufficient documentation (references available upon request). Four cases were judged to be wrongly diagnosed as PSR and were therefore excluded (40-43). Some old cases published by Japanese authors were also excluded because of sketchy data and the use of the term "nevus spilus" to indicate hypermelanotic patches regardless of the presence of superimposed speckles (44).

The two hallmark nevi were fully or predominantly ipsilateral in $83.3 \%$ of PSR cases and in $71.4 \%$ of PMR ones. Soft tissue and skeletal abnormalities, always ipsilateral to the NR, were present in $72.2 \%$ of PSR cases and in $21.4 \%$ of PMR ones. Neurological manifestations were found in five PSR cases (27.8\%), and cerebral 

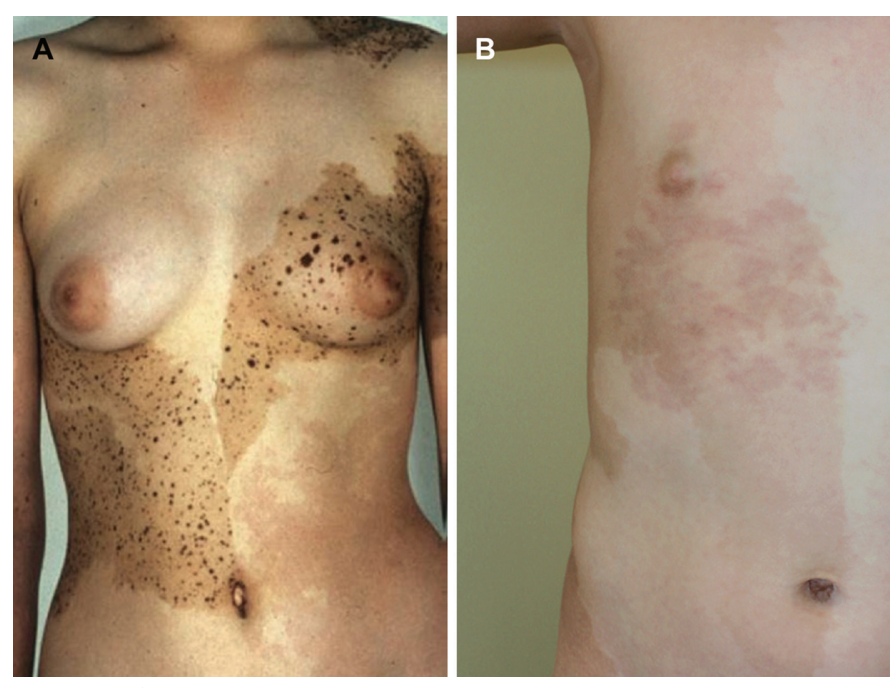

Figure $1 \mid$ A) Checkerboard-arranged macular nevus spilus and left-sided, lateralized nevus roseus (phacomatosis spilorosea; reprinted and modified with permission from Wiley [Happle R. J Eur Acad Dermatol. 2015;29:2295-305]); B) nevus roseus on the right side of the trunk superimposed on a large flag-like hypermelanotic nevus (phacomatosis melanorosea) (modified and reprinted from Tekin (39) under a Creative Commons Attribution-Noncommercial 4.0 International License).

Table 1 | Definite cases of phacomatosis spilorosea.

\begin{tabular}{|c|c|c|c|c|c|c|c|}
\hline $\begin{array}{l}\text { Reference } \\
\text { (number) }\end{array}$ & $\begin{array}{c}\text { Age } \\
\text { (years) }\end{array}$ & Sex & Ethnicity & $\begin{array}{c}\text { Lateralization of } \\
\text { nevus spilus }\end{array}$ & $\begin{array}{c}\text { Lateralization of } \\
\text { nevus roseus }\end{array}$ & $\begin{array}{c}\text { Main extracutaneous } \\
\text { abnormalities }\end{array}$ & $\begin{array}{c}\text { Additional } \\
\text { manifestations }\end{array}$ \\
\hline 8 & 11 & Female & White & $\begin{array}{l}\text { Left (head \& neck, } \\
\text { upper trunk, arm) }\end{array}$ & Right (trunk) & $\begin{array}{l}\text { Cerebral atrophy, } \\
\text { epilepsy, facial } \\
\text { nerve palsy, scoliosis }\end{array}$ & $\begin{array}{l}\text { Heterochromia iridis, } \\
\text { epidermal nevus, } \\
\text { Mongolian spot }\end{array}$ \\
\hline 9 & 26 & Female & White & Right (upper trunk, arm) & $\begin{array}{l}\text { Right (head \& neck, } \\
\text { upper trunk) }\end{array}$ & & \\
\hline 10 & 9 & Female & White & Right (trunk, arm) & Left (whole hemibody) & $\begin{array}{l}\text { Leg length discrepancy } \\
\text { (left > right), scoliosis }\end{array}$ & \\
\hline 11 & 18 & Male & White & Right (trunk, limbs) & Bilateral (limbs) & Lymphoedema (left leg) & \\
\hline 12 & 13 & Male & White & Right (trunk, arm) & Left (systematized) & $\begin{array}{l}\text { Sturge-Weber-Klippel- } \\
\text { Trenaunay phenotype (left } \\
\text { side) }\end{array}$ & \\
\hline 13 & 11 & Female & White & Bilateral (trunk, limbs) & Left (head \& neck, trunk) & $\begin{array}{l}\text { Leg length discrepancy } \\
\text { (left > right) } \\
\text { Facial nerve palsy }\end{array}$ & Granular cell tumors \\
\hline 14 & 21 & Female & White & $\begin{array}{l}\text { Left (head \& neck, } \\
\text { lower trunk, limbs) }\end{array}$ & Left (trunk, limbs) & $\begin{array}{l}\text { Leg length discrepancy } \\
\text { (left > right) }\end{array}$ & \\
\hline 15 & 16 & Male & White & $\begin{array}{l}\text { Left (head \& neck, } \\
\text { upper trunk, arm) }\end{array}$ & $\begin{array}{l}\text { Left (head \& neck, } \\
\text { upper trunk, arm) }\end{array}$ & & \\
\hline 16 & 38 & Female & White & $\begin{array}{l}\text { Bilateral (upper trunk, } \\
\text { arm) }\end{array}$ & $\begin{array}{l}\text { Bilateral (head \& neck, } \\
\text { upper trunk) }\end{array}$ & & \\
\hline 17 & 18 & Male & $\begin{array}{l}\text { Middle } \\
\text { Eastern }\end{array}$ & $\begin{array}{l}\text { Right (head \& neck, } \\
\text { upper trunk, arm) }\end{array}$ & Right (upper trunk) & & \\
\hline 18 & 5 & Female & White & $\begin{array}{l}\text { Left (head \& neck, } \\
\text { upper trunk, arm) }\end{array}$ & $\begin{array}{l}\text { Left (head \& neck, } \\
\text { upper trunk) }\end{array}$ & & Iris nodules \\
\hline 19 & 49 & Male & White & Right (lower trunk) & $\begin{array}{l}\text { Bilateral (head \& neck, } \\
\text { trunk, arms) }\end{array}$ & $\begin{array}{c}\text { Sturge-Weber-Klippel- } \\
\text { Trenaunay phenotype (right } \\
\text { side) }\end{array}$ & \\
\hline 20 & 13 & Male & White & Right (head \& neck) & $\begin{array}{l}\text { Bilateral (head \& neck, } \\
\text { upper trunk, arms) }\end{array}$ & $\begin{array}{l}\text { Klippel-Trenaunay } \\
\text { phenotype (left side) }\end{array}$ & \\
\hline 21 & 11 & Female & White & Left (whole hemibody) & Bilateral (whole body) & $\begin{array}{l}\text { Leg length discrepancy } \\
\text { (right > left) }\end{array}$ & \\
\hline 22 & 12 & Female & White & Right (whole hemibody) & Right (whole hemibody) & Lymphoedema (right leg) & \\
\hline 23 & 7 & Male & Amerindian & $\begin{array}{l}\text { Left (head \& neck, } \\
\text { trunk, arm) }\end{array}$ & Left (upper trunk, limbs) & $\begin{array}{l}\text { Leg length discrepancy } \\
\text { (left > right), scoliosis }\end{array}$ & Oligodontia \\
\hline 24 & 12 & Female & White & $\begin{array}{l}\text { Right (head \& neck, } \\
\text { trunk, arm) }\end{array}$ & Right (whole hemibody) & $\begin{array}{l}\text { Leg length discrepancy } \\
\text { (right > left), scoliosis } \\
\text { Macrocephaly, } \\
\text { developmental delay, } \\
\text { muscular hypotonia }\end{array}$ & Irregular astigmatism \\
\hline 25 & 10 & Male & White & $\begin{array}{l}\text { Left (head \& neck, } \\
\text { upper trunk) }\end{array}$ & Left (whole hemibody) & $\begin{array}{l}\text { Leg length discrepancy } \\
\text { (left > right), lymphedema }\end{array}$ & \\
\hline
\end{tabular}

vascular anomalies were detected in two PMR instances $(32,34)$. Additional cutaneous lesions were present mostly in PMR (35.7\% of cases). No extracutaneous abnormalities were reported in four cases of PSR (22.2\%) and in eight cases of PMR (57.1\%), often reflecting limited cutaneous involvement.

\section{Discussion}

This critical review makes it possible to clear the field of inappropriately labeled cases and to establish sine dubio cases of PSR and PMR published in the worldwide literature. Of note, six previously unrecognized PMR cases emerged (26-29, 33, 34), including four in which the diagnosis was redirected from that of PSR $(27-29,34)$. A diagnosis of PSR was retrospectively achieved in one instance (12), and another PSR case was finally brought to the attention of mainstream literature (17).

In terms of clinical associations, some differences between the two entities emerged. In fact, PSR predominantly involves the musculoskeletal system and can be complicated by neurological manifestations. In contrast, PMR is sometimes associated with ancillary cutaneous lesions; displays a relevant association with vascular malformations of the brain (although its frequency might

\section{Main extracutaneous Addional}


be lower than previously thought [4]), and in general appears to represent a less severe syndrome.

Unlike the PPV types associated with NC, which in all likelihood prevalently involve East Asians and Amerindians (5, 45), whites seem to be the predominantly affected ethnic group, notwithstanding, as mentioned above, the unconfirmed nature of half a dozen early Japanese reports (44). Besides the defining nevi, the main differential feature between NC-associated PPVs and NR-associated PPVs seems to be ocular manifestations in the former (glaucoma and choroidal melanoma), which are to be attributed to the occurrence of nevus of Ota (5).

Two recent reports provided evidence for consideration of two additional PPV variants consisting of more than two hallmark nevi ("phacomatosis multiplex"), that is, phacomatosis cesioflammeomarmorata (46) and phacomatosis melanocesioflammea (47).

These reappraisals of the literature leave only a handful of still unclassifiable PPV cases. Two cases have been reported, featuring the association of flag-like hypomelanotic nevus and nevus flammeus $(48,49)$. A case studied by Bielsa et al., consisting of a systematized MNS associated with nevus anemicus and bilateral leg lymphedema, could be an additional example of PSR (50). In fact, it is possible that an NR might have gone unnoticed due to its pale hue and mazy intermingling with nevus anemicus. A patient mentioned as part of a PPV case series was categorized as "unclassifiable" but might actually be another PMR instance (51). A case labeled with a diagnosis of "phacomatosis achromico-melano-marmorata" raises doubts due to a number of sketchy clinical features (52). Another patient featured a combination of phacomatosis cesioflammea and nevus vascularis mixtus (53). Finally, it is worth mentioning two patients that showed a combination of pink telangiectatic nevus, nevus anemicus, and FHN (thereby displaying overlapping features of mixed vascular nevus syndrome and PMR) together with vascular malformations of the brain and ipsilateral hypotrophy $(54,55)$. Therefore, with the possible exception of the association of flag-like hypomelanotic nevus and nevus flammeus $(48,49)$, no other PPV variants still waiting to be unearthed can be foreseen according to current evidence.

Based on the above considerations, and the fact that a causative postzygotic gene mutation has been identified in some instances $(56,57)$, an updated classification of PPV can be suggested, which includes six definite types (Table 3). Future clinical and molecular research will hopefully help further substantiate and expand the view of the established PPV variants as distinctive clinicogenetic entities.

Table 2 | Definite cases of phacomatosis melanorosea.

\begin{tabular}{|c|c|c|c|c|c|c|c|}
\hline $\begin{array}{l}\text { Reference } \\
\text { (number) }\end{array}$ & $\begin{array}{c}\text { Age } \\
\text { (years) }\end{array}$ & Sex & Ethnicity & $\begin{array}{c}\text { Lateralization of } \\
\text { nevus spilus }\end{array}$ & $\begin{array}{c}\text { Lateralization of } \\
\text { nevus roseus }\end{array}$ & $\begin{array}{c}\text { Main extracutaneous } \\
\text { abnormalities }\end{array}$ & $\begin{array}{c}\text { Additional } \\
\text { manifestations }\end{array}$ \\
\hline 26 & 22 & Female & East Asian & Bilateral (systematized) & Bilateral (systematized) & & \\
\hline 27 & 11 & Female & East Asian & Bilateral (legs) & Right (trunk, arm) & $\begin{array}{l}\text { Leg length discrepancy } \\
\text { (right }>\text { left) }\end{array}$ & \\
\hline 28 & 21 & Male & White & $\begin{array}{l}\text { Left (head \& neck, } \\
\text { upper trunk, arm) }\end{array}$ & $\begin{array}{l}\text { Right (head \& neck, } \\
\text { lower trunk, limbs) }\end{array}$ & & \\
\hline 29 & 15 & Female & East Asian & Right (whole hemibody) & Right (limbs) & $\begin{array}{l}\text { Leg length discrepancy } \\
\text { (right }>\text { left) }\end{array}$ & \\
\hline 30 & 2 & Male & White & $\begin{array}{l}\text { Right (head \& neck, } \\
\text { trunk, leg) }\end{array}$ & $\begin{array}{l}\text { Right (upper trunk, } \\
\text { limbs) }\end{array}$ & $\begin{array}{l}\text { Epilepsy, small cerebral } \\
\text { lesions }\end{array}$ & Iris nodules \\
\hline 31 & 8 & Female & White & Right (head \& neck) & Right (head \& neck) & & Achromic lesions \\
\hline 32 & 1 & Male & East Asian & Left (trunk, leg) & $\begin{array}{l}\text { Left (head \& neck, } \\
\text { upper trunk) }\end{array}$ & Moyamoya & \\
\hline 33 & 7 & Female & White & Left (lower limb) & Right (lower limb) & & \\
\hline 34 & 20 & Male & White & $\begin{array}{l}\text { Right (head \& neck, } \\
\text { upper trunk, arm) }\end{array}$ & $\begin{array}{l}\text { Left (head \& neck, } \\
\text { upper trunk, arm) }\end{array}$ & $\begin{array}{c}\text { Venous anomaly } \\
\text { of brain }\end{array}$ & $\begin{array}{c}\text { Becker nevus, } \\
\text { agminated lentigines }\end{array}$ \\
\hline 35 & 9 & Female & White & Left (lower trunk, leg) & Left (head \& neck, leg) & & \\
\hline 36 & 2 & Female & White & Bilateral (trunk, arm) & Right (trunk, limbs) & Hypertrophy (right limbs) & \\
\hline 37 & 11 & Female & White & Left (trunk, limbs) & $\begin{array}{c}\text { Bilateral } \\
\text { (whole hemibody) }\end{array}$ & & $\begin{array}{l}\text { Heterochromia of } \\
\text { scalp hair }\end{array}$ \\
\hline 38 & 5 & Female & White & Right (leg) & Left (leg) & & \\
\hline 39 & 3 & Male & White & Right (trunk, leg) & Right (head \& neck, trunk, arm) & & Agminated lentigines \\
\hline
\end{tabular}

Table 3 | Updated classification of phacomatosis pigmentovascularis.

\begin{tabular}{lcc}
\hline Type & Pigmented nevus/i & Capillary nevus/i \\
\hline Phacomatosis cesioflammea & Nevus cesius & Nevus flammeus \\
Phacomatosis cesiomarmorata & Nevus cesius & Cutis marmorata telangiectatica congenita \\
Phacomatosis spilorosea & Macular nevus spilus & Nevus roseus \\
Phacomatosis melanorosea & GNA1156 & Nevus roseus \\
Phacomatosis cesioflammeomarmorata & Flag-like hypermelanotic nevus & Nevus flammeus, \\
Phacomatosis melanocesioflammea & Nevus cesius & Unknown \\
& Flag-like hypermelanotic nevus, & Unknown \\
& Nevus cesius & Nevus flammeus
\end{tabular}

\section{References}

1. Ota M, Kawamura T, Ito N. [Phacomatosis pigmentovascularis (Ota)]. Jpn J Dermatol. 1947;52:1-3. Japanese.

2. Torrelo A, Zambrano A, Happle R. Cutis marmorata telangiectatica congenita and extensive Mongolian spots: type 5 phacomatosis pigmentovascularis. $\mathrm{Br}$ Dermatol. 2003;148:342-5.
3. Happle R. Phacomatosis pigmentovascularis revisited and reclassified. Arch Dermatol. 2005;141:385-8.

4. Torchia D. Phacomatosis melanorosea, unearthed. Eur J Dermatol. 2012;22:5823. 
5. Kumar A, Zastrow DB, Kravets EJ, Beleford D, Ruzhnikov MRZ, Grove ME, et al. Extracutaneous manifestations in phacomatosis cesioflammea and cesiomarmorata: case series and literature review. Am J Med Genet A. 2019;179:966-77.

6. Bui TN, Corap A, Bygum A. Cutis marmorata telangiectatica congenita: a literature review. Orphanet J Rare Dis. 2019;14:283.

7. Torchia D, Happle R. Segmental hypomelanosis and hypermelanosis arranged in a checkerboard pattern are distinct naevi: flag-like hypomelanotic naevus and flag-like hypermelanotic naevus. J Eur Acad Dermatol Venereol. 2015;29:208899.

8. Westerhof W, Fleury P, Smitt J. Phakomatosis pigmentovascularis type IVB. Br J Dermatol. 1986;115:589-90.

9. Zahorcsek Z, Schmelás A, Schneider I. Progrediente zirkumskripte Lentiginose Phakomatosis pigmentovascularis III/A. [Progressive circumscript lentiginosisphakomatosis pigmentovascularis III/A]. Hautarzt. 1988;39:519-23. German.

10. Sigg C, Pelloni F. Oligosymptomatic form of Klippel-Trenaunay-Weber syndrome associated with giant nevus spilus. Arch Dermatol. 1989;125:1284-5.

11. Happle R, Steijlen PM. Phacomatosis pigmentovascularis gedeutet als ein Phänomen der Zwillingsflecken. [Phacomatosis pigmentovascularis interpreted as a phenomenon of twin spots]. Hautarzt. 1989;40:721-4. German.

12. Stevanović D, Minić S, Glavicić M, Krunić A, Djaković Z. Phacomatosis pigmentovascularis: a new association. Br J Dermatol. 1991;125:86.

13. Guiglia MC, Prendiville JS. Multiple granular cell tumors associated with giant speckled lentiginous nevus and nevus flammeus in a child. J Am Acad Dermatol. 1991;24:359-63.

14. Libow LF. Phakomatosis pigmentovascularis type IIIb. J Am Acad Dermatol. 1993;29:305-7.

15. Mahroughan M, Mehregan AH, Mehregan DA. Phakomatosis pigmentovascularis: report of a case. Pediatr Dermatol. 1996;13:36-8.

16. Stadhouders-Keet SA, Glastra A, Van Vloten WA. Phakomatosis pigmentovascularis (type IIIa). [Phakomatosis pigmentovascularis (type IIla)]. Ned Tijdschr Geneeskd. 1999;143:1337. Dutch.

17. Farshchian M, Nabi Zadeh K. [Phakomatosis pigmentovascularis: a case report]. Iran J Dermatol. 2000;3:42-5. Persian.

18. Ruiz Villaverde R, Blasco Melguizo J, Martin Sanchez MC. Facomatosis pigmentovascular tipo IIla. [Phacomatosis pigmentovascularis type IIIa]. Rev Esp Pediatr. 2002;58:295-97. Spanish.

19. Diociaiuti A, Guidi B, Aguilar Sanchez JA, Feliciani C, Capizzi R, Amerio P. Phakomatosis pigmentovascularis type IIIb: a case associated with Sturge-Weber and Klippel-Trenaunay syndromes. J Am Acad Dermatol. 2005;53:536-9.

20. Borlu M, Uksal U, Utas S, Ferahbas A. Phakomatosis pigmentovascularis typeIIIb with Klippel-Trenaunay-Weber syndrome. Eur J Pediatr Dermatol. 2007;17: 31-4.

21. Seckin D, Yucelten D, Aytug A, Demirkesen C. Phacomatosis pigmentovascularis type IIIb. Int J Dermatol. 2007;46:960-3.

22. Jordaan HF, Happle R. Phacomatosis spilorosea associated with lymphoedema. Br J Dermatol. 2008;159:489-91.

23. Valdivielso-Ramos M, Mauleón C, Hernanz JM. Phacomatosis spilorosea with oligodontia, scoliosis and fibrous cortical defects. J Eur Acad Dermatol Venereol. 2012;26:260-2.

24. Fink C, Happle R, Enk A, Haenssle HA. Phacomatosis spilorosea: visual diagnosis and associated pathologies of a rare entity. J Eur Acad Dermatol Venereol. 2016;30:e69-70.

25. Convalexius P, Luger TA, Böhm M. Kongenitale Gefäß- und Pigmentmale mit Lymphödem, Beinlängendifferenz und Skoliose. [Congenital telangiectatic und pigmented lesions associated with lymphedema, difference in leg length, and scoliosis]. J Deutsch Dermatolog Gesellsch. 2017;15:751-4. German.

26. Toda K. [Two cases of a new type of phacomatosis pigmentovascularis Ota]. Jpn J Dermatol A. 1966;76:47-51. Japanese.

27. Hasegawa Y, Yasuhara M. [A case of phacomatosis pigmentovascularis type IIIb]. Skin Res. 1990;32:71-81. Japanese.

28. Prigent F, Kahn A, Martinet C, Saigot T. Phakomatose pigmento-vasculaire IIla. [Phacomatosis pigmentovascularis IIIa]. Ann Dermatol Venereol. 1991;118:5313. French.

29. Choi JY, Lee MH, Haw CR. [A case of phakomatosis pigmentovascularis associated with leg-length discrepancy]. Korean J Dermatol. 1994;32:707-12. Korean.

30. Romiti R, Nico MMS, Rezende MB, Cresta FB, Sotto MN, Rivitti EA. Child with macular hyperpigmentation, nevus flammeus, and systemic disorders: a variant of phakomatosis pigmentovascularis? Eur J Pediatr Dermatol. 1997;7:93-8.

31. de las Heras E, Boixeda JP, Ledo A, Happle R. Paired melanotic and achromic macules in a case of phacomatosis pigmentovascularis: a further example of twin spotting? Am J Med Genet. 1997;70:336-7.

32. Tsuruta D, Fukai K, Seto M, Fujitani K, Shindo K, Hamada T, et al. Phakomatosis pigmentovascularis type IIIb associated with moyamoya disease. Pediatr Dermatol. 1999;16:35-8.
33. Gómez Bezares P, Vázquez Doval FJ. Facomatosis pigmentovascular. [Phacomatosis pigmentovascularis]. Pediatría rural y extrahospitalaria. 2006;36:35-7. Spanish.

34. Karabudak O, Dogan B, Basekim C, Harmanyeri Y. Phacomatosis spilorosea (phacomatosis pigmentovascularis type IIIb). Australas J Dermatol. 2007;48:256-8.

35. Garofalo L. Phakomatosis pigmento-vascularis. Eur J Pediatr Dermatol. 2011;21: 248.

36. Aguayo-Leiva I, Alonso J, Echeverría B, Hernández-Martín A, Torrelo A. Phacomatosis melanovascularis: a new example of non-allelic twin spotting. Eur J Dermatol. 2011;21:487-9.

37. Almeida H Jr, Happle R Jr, Reginatto F Jr, Basso F Jr, Duquia R Jr. Phacomatosis melanorosea with heterochromia of scalp hair. Eur J Dermatol. 2011;21:598-9.

38. Arnold AW, Kleine MU, Happle R. Phacomatosis melanorosea without extracutaneous features: an unusual type of phacomatosis pigmentovascularis. Eur J Dermatol. 2012;22:473-5.

39. Tekin B, Yucelten D, Happle R. Phacomatosis melanorosea: a further case of an unusual skin disorder. Acta Derm Venereol. 2016;96:280-2.

40. Suzuki K, Ishizaki H, Takahashi H. [Phacomatosis pigmentovascularis IIlb associated with porokeratosis, acanthosis nigricans and endocrinopathy in brother and sister]. Skin Res. 1990;32:65-70. Japanese.

41. Hidalgo Dominguez R, Perez Moyano R, Toribio Onieva JR, Garcia Mellado V, Tercedor J. Type IIla pigmented vascular phacomatosis. [Type IIla phacomatosis pigmentovascularis]. Actas dermo-sifiliográficas 1994;85:349-51. Spanish.

42. Ruiz Villaverde R, Viera Ramirez A, Linares Solano J, Naranjo Sintes R, Gutierrez Salmerón MT. Phakomatosis pigmentovascularis and Lisch nodules. Relationship between Von Recklinghausen and phakomatosis pigmentovascularis? J Eur Acad Dermatol Venereol. 2003;17:53-5.

43. Fischer K, Trautinger F. Phacomatosis pigmentovascularis. [Phacomatosis pigmentovascularis]. J Deutsch Dermatolog Gesellsch. 2015;1311:1178-9. German.

44. Hasegawa Y, Yasuhara M. Phakomatosis pigmentovascularis type IVa. Arch Dermatol. 1985;121:651-5.

45. Vidaurri-de la Cruz H, Tamayo-Sánchez L, Durán-McKinster C, Orozco-Covarrubias ML, Ruiz-Maldonado R. Phakomatosis pigmentovascularis Ila and IIb: clinical findings in 24 patients. J Dermatol. 2003;30:381-8.

46. Chehad AS. New case of phacomatosis cesio-flammeo-marmorata: the time is right to review the classification for phacomatosis pigmentovascularis. Int J Dermatol. 2019;58:e237-40.

47. Torchia D. Phacomatosis melanocesioflammea: the rediscovery of phacomatosis pigmentovascularis type IV. Int J Dermatol. 2020;59:e381-3.

48. Dippel E, Utikal J, Feller G, Fackel N, Klemke CD, Happle R, et al. Nevi flammei affecting two contralateral quadrants and nevus depigmentosus: a new type of phacomatosis pigmentovascularis? Am J Med Genet A. 2003;119A:228-30.

49. Elmas OF, Kızılyel O, Metin MS, Bilen H, Atasoy M. Nevüs flammeus ve nevüs depigmentozus birlikteliği: yeni bir fakomatozis pigmentovaskülaris tipi mi? [Coexistence of nevus flammeus and nevus depigmentosus: is it a new subtype of phakomatosis pigmentovascularis?]. Türkderm. 2015;49:167-8. Turkish.

50. Bielsa I, Paradelo C, Ribera M, Ferrándiz C. Generalized nevus spilus and nevus anemicus in a patient with a primary lymphedema: a new type of phakomatosis pigmentovascularis? Pediatr Dermatol. 1998;15:293-5.

51. Fernández-Guarino M, Boixeda P, de Las Heras E, Aboin S, García-Millán C, Olasolo PJ. Phakomatosis pigmentovascularis: clinical findings in 15 patients and review of the literature. J Am Acad Dermatol. 2008;58:88-93.

52. Boente MC, Obeid R, Asial RA, Bibas-Bonet H, Coronel AM, Happle R. Cutis tricolor coexistent with cutis marmorata telangiectatica congenita: "phacomatosis achromico-melano-marmorata." Eur J Dermatol. 2008;18:394-6.

53. Hayashi S, Kaminaga T, Tantcheva-Poor I, Hamasaki Y, Hatamochi A. Patient with extensive Mongolian spots, nevus flammeus and nevus vascularis mixtus: a novel case of phacomatosis pigmentovascularis. J Dermatol. 2016;43:225-6.

54. Hidano A, Arai Y. Hémihypertrophie congénitale associée a des anomalies cutanées pigmento-vasculaires, cérébrales, viscérales et squelettiques. [Congenital hemihypertrophy associated with cutaneous pigmento-vascular, cerebral, visceral and bone abnormalities]. Ann Dermatol Venereol. 1987;114:665-9. French.

55. Toelle SP, Weibel L, Schiegl H, Boltshauser E. Phacomatosis pigmentovascularis and extensive venous malformation of brain vessels: an unknown association or a new vascular neurocutaneous syndrome? Neuropediatrics. 2011;42:234-6.

56. Thomas AC, Zeng Z, Rivière JB, O'Shaughnessy R, Al-Olabi L, St-Onge J, et al. Mosaic activating mutations in GNA11 and GNAQ are associated with phakomatosis pigmentovascularis and extensive dermal melanocytosis. J Invest Dermatol. 2016;136:770-8.

57. Polubothu S, Bohm M, Fink C, Haenssle H, Happle R, Patton E, et al. Phakomatosis pigmentovascularis spilorosea and speckled lentiginous naevus syndrome are caused by mosaic mutations in gene PTPN11. Pediatr Dermatol. 2019;36:S7. 